\title{
Polymer-based tubular microbots: role of composition and preparation $\dagger$
}

\author{
Wei Gao, Sirilak Sattayasamitsathit, Aysegul Uygun, Allen Pei, Adam Ponedal and Joseph Wang* \\ Received 16th January 2012, Accepted 14th February 2012 \\ DOI: 10.1039/c2nr30138e
}

The influence of the composition and electropolymerization conditions upon the propulsion of new template-prepared polymer-based bilayer microtubular microbots is described. The effects of different electropolymerized outer layers, including polypyrrole (PPy), poly(3,4-ethylenedioxythiophene) (PEDOT), polyaniline (PANI), and of various inner catalytic metal surfaces (Ag, Pt, Au, Ni-Pt alloy), upon the movement of such bilayer microtubes are evaluated and compared. Electropolymerization conditions, such as the monomer concentration and medium (e.g. surfactant, electrolyte), have a profound effect upon the morphology and locomotion of the resulting microtubes. The most efficient propulsion is observed using PEDOT/Pt microbots that offer a record-breaking speed of over 1400 body lengths $\mathrm{s}^{-1}$ at physiological temperature, which is the fastest relative speed reported to date for all artificial micro/nanomotors. An inner Pt-Ni alloy surface is shown useful for combining magnetic control and catalytic fuel decomposition within one layer, thus greatly simplifying the preparation of magnetically-guided microbots. Polymer-based microbots with an inner gold layer offer efficient biocatalytic propulsion in low peroxide level in connection to an immobilized catalase enzyme. Metallic $\mathrm{Au} / \mathrm{Pt}$ bilayer microbots can also be prepared electrochemically to offer high speed propulsion towards potential biomedical applications through functionalization of the outer gold surface. Such rational template preparation and systematic optimization of highly efficient microbots hold considerable promise for diverse practical applications.

\section{Introduction}

Inspired by the sophistication of nature biomotors and driven by recent nanotechnological advances, major efforts are currently being devoted to the design of efficient synthetic micro/nanoscale motors that convert various energies into autonomous motion, ${ }^{1-15}$ especially to catalytic motors based on the decomposition of hydrogen peroxide. ${ }^{1-7}$ Among all the catalytic nanomotors, the tubular microengines - pioneered by Mei and Schmidt - are particularly attractive and powerful for diverse practical applications. ${ }^{16-19}$ The oxygen-bubble propulsion mechanism of tubular microengines leads to efficient locomotion in relevant biological fluids and high ionic-strength media, as desired for different biomedical applications. ${ }^{20-24}$ While offering extremely attractive performance, the practical utility of rolledup microengines is hindered by the complexity of the fabrication process and related (clean-room) costs. A greatly simplified template-membrane based synthesis of highly efficient and smaller microtube engines was reported recently. ${ }^{25}$ The new template electrodeposition protocol has led to ultrafast (>350 body lengths $\left.\mathrm{s}^{-1}\right)$ PANI/Pt bilayer tubular microbots $(8-\mu \mathrm{m}$

Department of Nanoengineering, University of California San Diego, San Diego, CA 92093, USA. E-mail: josephwang@ucsd.edu; Fax: +1-858534-9553; Tel: +1-858-246-0128

$\dagger$ Electronic supplementary information (ESI) available: Supporting Figures and videos. See DOI: 10.1039/c2nr30138e long), requiring very low fuel concentrations (down to $0.2 \%$ $\mathrm{H}_{2} \mathrm{O}_{2}$ ). ${ }^{25}$ Such ultrafast nanomotor speed reflects a large force and power essential to execute different tasks.

Here we report on a critical evaluation of template-prepared polymer-metal tubular microbots based on different materials and synthesis conditions and on the dramatically improved propulsion performance achieved by identifying new compositions and optimizing their preparation protocol. We indicate that when polymer-based microstructures are prepared by templatedirected electrochemical synthesis, there is an opportunity to control the morphology. In particular, we critically compare microbots based on different electropolymerized outer layers and various metallic and alloy inner layers. As excepted for template directed electrochemical synthesis, the preparation conditions, particularly the electropolymerization conditions, are shown to have a profound effect upon the morphology and propulsion behavior. The movement is also shown to be greatly influenced by the choice of monomer structure that affects the diameter and shape. This detail study has thus led to useful insights into the preparation and composition of high-performance biocompatible low-cost template-prepared tubular microbots and facilitated the rational design of ultrafast PEDOT/Pt bilayer microbots with a record-breaking speed of over 1400 body lengths per second. In addition to pursuit the ultrafast propulsion, we also describe the first example of microbots based on an inner Pt-Ni alloy surface combining (within the same layer) 
catalytic and magnetic properties for the peroxide decomposition and magnetic alignment, respectively. Bimetallic Au-Pt microbots are also presented here for the first time, with the presence of a gold outer layer facilitating surface functionalization towards potential biomedical applications.

Particular attention is given to the role of the electropolymerized outer layer and to the influence of different inner catalytic layers. Compared to common metallic materials, conducting polymers, such as polyaniline (PANI), polypyrrole (PPy), polythiophene (PT) and poly(3,4-ethylenedioxythiophene) (PEDOT) offer considerable promise for various practical applications due to their light weight, conductivity, mechanical flexibility, unique chemical and redox properties, along with minimal structural defects, high aqueous stability and biocompability. ${ }^{26-32}$ Functionalized-polymer microstructures have thus found a growing role as microscale actuators, drug carriers or metabolite and genetic biosensors. ${ }^{33-35}$ For sensing applications of conducting polymers, the tubular forms of conducting polymers have been shown advantageous (over film, fibrillar or wire forms) owing to their high surface area and fast response. ${ }^{36}$ Conducting polymer nanotubes, suitable for self-propelled tubular microbots, can be prepared by both oxidative-chemical, soft-template and hard-template electrochemical polymerization methods; although little attention has been given to electrochemical preparation of conducting-polymer microtubes, the electropolymerization route is preferred since it offers precise control of the deposition conditions and hence of the dimensions and morphology of the resulting microtubes..$^{37,38}$ The exact microstructures and morphologies of the resulting conducting polymers have a profound effect on their physical and chemical properties. ${ }^{39,40}$ Here we investigate the preparation of self-propelled microbots based on different conducting polymers and electropolymerization conditions (e.g., monomer, surfactant, supporting electrolyte and monomer concentration). The influence of these conditions upon the structure and behavior of the resulting microbots is investigated in connection to PPy-, PEDOT- and PANI-based microtubes. The systematic optimization of the composition and preparation of such polymer-based tubular microbots, reported in the following sections, offers useful insights towards the rational preparation of high-performance microbots with distinct advantages over existing microscale motors.

\section{Results and discussions}

Various polymer-based bilayer microtubes have been successfully fabricated in this study using template-electrodeposition method in connection to a Cyclopore polycarbonate membrane, with conical-shaped micropores. The nucleation and growth of conducting polymer microtubes involve electrostatic and solvophobic interactions between the polymers and pore wall. ${ }^{37}$ Poly(3,4-ethylenedioxythiophene) (PEDOT), polyaniline (PANI) and polypyrrole (PPy) were selected for the present microbot work owing to their inherent chemical stability ${ }^{41}$ As expected and will be illustrated below, the thickness (and hence opening side) and the length of the polymer-based microtubes depends on the synthesis conditions. The most favorable electrochemical growth conditions of PEDOT, PANI and PPy (described below) were optimized for their microtubes bilayer

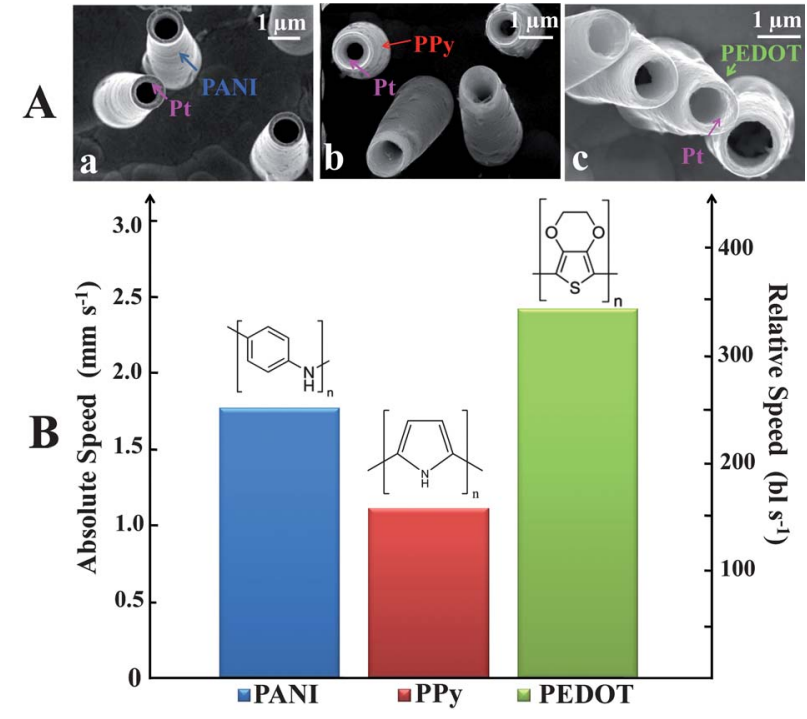

Fig. 1 A) SEM images of polymer-based template growth of the bilayer microtubes: a) PANI/Pt; b) PPy/Pt; c) PEDOT/Pt. B) The absolute and relative speeds of the polymer-based bilayer microtubes in a $5 \% \mathrm{H}_{2} \mathrm{O}_{2}$ solution containing $2.67 \%$ sodium cholate.

structures. Fig. 1A displays the SEM images of the three different template-prepared bilayer microtubes under the optimized conditions: PANI/Pt (a), PPy/Pt (b) and PEDOT/Pt (c). In general, such bilayer microtubes were prepared following the previously described method. ${ }^{25}$ PPy and PEDOT microtubes were electrosynthesized at $+0.8 \mathrm{~V}$ using $7.5 \mathrm{mM} \mathrm{KNO}_{3}$ as the electrolyte, and charges of $0.8 \mathrm{C}$ and $0.06 \mathrm{C}$, respectively; PANI microtube was prepared using the same potential in a plating solution containing $0.1 \mathrm{M} \mathrm{H}_{2} \mathrm{SO}_{4}, 0.5 \mathrm{M} \mathrm{Na}_{2} \mathrm{SO}_{4}$ and $0.1 \mathrm{M}$ aniline for a charge of $0.02 \mathrm{C}$. Subsequently, the inner Pt layer was deposited galvanostatically at $-2 \mathrm{~mA}$ for $600 \mathrm{~s}$ from a commercial platinum plating solution. The presence of $\mathrm{HSO}_{4}$ anion, acidic media, and sodium cations provides a high PANI polymerization rate, conductivity and electroactivity. ${ }^{41,42}$ The electropolymerization of PEDOT commonly involves plating solutions containing the sodium dodecyl sulfate (SDS) surfactant for improving the monomer solubility in aqueous solution and lowering the oxidation potentia ${ }^{43,44}$ resulting in improved the opening size and surface morphology of the microtubes. The images of Fig. 1, involving these 3 different monomers, clearly indicate that the template electrodeposition method leads to bilayer microtubes with uniform morphology. The resulting bilayer PANI/Pt (a), PPy/Pt (b) and PEDOT/Pt (c) microtubes ( $\sim 7 \mu \mathrm{m}$ long) are characterized with different front opening diameters of $0.8 \mu \mathrm{m}, 0.6 \mu \mathrm{m}$ and $1 \mu \mathrm{m}$, respectively. Such different openings can be attributed to differences in the sizes of the polymer chains, electropolymerization rate and in the packing patterns of the different polymers. ${ }^{45}$ The morphological features of the polymer films have a profound effect upon their electrochemical and electrochromic properties. ${ }^{46}$ Comparing the formation of PEDOT, PANI and PPy structures, it can be observed that the PANI and PEDOT tubules have larger opening and do not close up, even after a long polymerization time. In contrast, the PPy tubules usually have a thicker layer, and form wires or fibers after long polymerization time, filling the pores 
completely. ${ }^{47}$ EDX mapping analysis, shown in SI Fig. 1, confirmed the presence of $\mathrm{Pt}, \mathrm{N}$ of the PPy/Pt (a) and $\mathrm{Pt}, \mathrm{S}$ of the $\mathrm{PEDOT} / \mathrm{Pt}$ (b), and the successful growth of the bilayer microtubes.

Fig. 1B compares the speeds of the various polymer-based microbots (in the presence of $5 \%$ hydrogen peroxide along with $2.67 \%$ (w/v) sodium cholate as surfactant). The PPy/Pt, PEDOT/ $\mathrm{Pt}$ and PANI/Pt bilayer microbots display ultrafast speeds of 1120, 2400 and $1700 \mu \mathrm{m} \mathrm{s}^{-1}$ (corresponding to 160, 340 and 240 body lengths $\mathrm{s}^{-1}$ ), respectively (See SI Video 1). Such differences in the speed are attributed primarily to the size of the opening diameter. ${ }^{17,25,48}$ Considering the tubular microbot as a cylinder microrod (since the fluid cannot freely flow through microbot because of the oxygen bubbles), the fluid drag coefficient is expected to be the same for the microbots with the same outer diameter and different inner opening diameters, ${ }^{25,48}$ however, the larger inner opening is coupled with a larger catalytic surface area, hence facilitating the bubble evolution and leading to a faster speed. ${ }^{48}$ Additional experiments using a higher $(10 \%)$ peroxide level showed that the PEDOT/Pt microbots can achieve an ultrafast average speed of $3350 \mu \mathrm{m} \mathrm{s}^{-1}$ (around 480 body lengths $\mathrm{s}^{-1}$ ). As will be illustrated below, even further acceleration and a new speed record (of 1400 body lengths $\mathrm{s}^{-1}$ ) can be achieved by operating these PEDOT/Pt microbots at a physiological temperature. It should also be pointed out that the preparation of the PEDOT-based bilayer microtubes provides more reproducible yields and consistent batch-to-batch morphology and length, especially compared to fast growing PANI-based tubular microengines (involving a very rapid aniline electropolymerization). ${ }^{25}$ Overall, PEDOT-based miccrobots provide the most favorable preparation and propulsion performance.

Scanning electron microscope (SEM) images show useful insights into the morphological features of PEDOT/Pt tubular microbots, grown in the different synthesis media. As indicated from the images of Fig. 2(a-d), the monomer concentration and
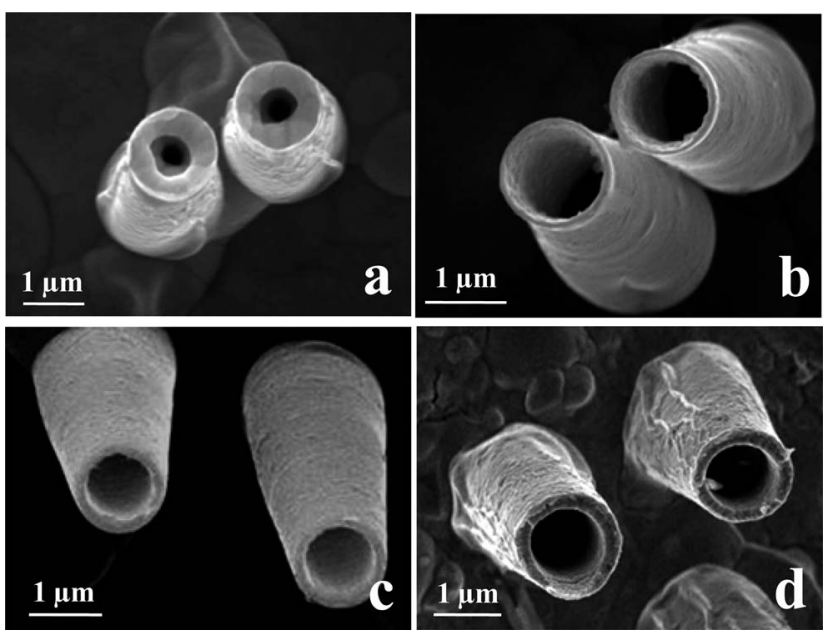

Fig. 2 The SEM images of PEDOT based bilayer microtube prepared under different conditions: a) $100 \mathrm{mM}$ EDOT and $100 \mathrm{mM}$ SDS; b) 15 $\mathrm{mM}$ EDOT and $100 \mathrm{mM} \mathrm{SDS}$; c) $15 \mathrm{mM}$ EDOT and $2 \mathrm{mM}$ SDS; d) 15 $\mathrm{mM}$ EDOT alone. Supporting electrolyte (during the electropolymerization): $7.5 \mathrm{mM} \mathrm{KNO}_{3}$. the presence of surfactant in the electropolymerization media have a profound effect upon the morphology and opening diameter of the resulting polymer microtubes. The effect of the monomer concentration was investigated by comparing morphology of the microbots in electrochemical template systems using 15 and $100 \mathrm{mM}$ EDOT (in the presence of $100 \mathrm{mM}$ SDS). The higher monomer concentration led to less uniform, rougher tubular structures with thicker walls (i.e., smaller opening pore) (Fig. 2, a vs. b). It can be concluded that solvophobic and electrostatic interactions between the pore wall of membrane and reacting species can result in the preferential nucleation and growth of PEDOT onto the pores of the membrane wall, producing microtube structure with a thicker polymer layer. ${ }^{49}$ PEDOT/Pt microtubes prepared using $15 \mathrm{mM}$ EDOT solution are characterized with a smoother more regular surface morphology, along with thinner walls. Such improved microtube structures containing a wider opening pore, lead to faster speed compared with PEDOT/Pt microbots prepared in presence of higher monomer concentration.

The surfactant effect was also examined for the preparation of PEDOT/Pt microbots using the same monomer concentration. Due to the low solubility of EDOT in water, the electropolymerization of EDOT was usually carried out in organic solvents (which may deteriorate the polycarbonate membrane pores). ${ }^{50}$ Recently, the surfactants were used to control the morphology of the PEDOT film with electrochemical methods in an aqueous solution. ${ }^{51}$ Herein, the surfactant effect upon the PEDOT/Pt microtube structures was investigated using plating solutions containing different surfactant concentrations (2 and $100 \mathrm{mM}$ ) and $15 \mathrm{mM}$ of the EDOT monomer. The film thickness increased (from 100 to $150 \mathrm{~nm}$ ) upon decreasing the surfactant concentrations from 100 to $2 \mathrm{mM}$ (Fig. 2(b) and 2(c)). Without the surfactant, the polymerization created a non-uniform and thicker polymer layer, displayed in Fig. 2(d), leading to a smaller inner diameter which greatly hinders the microbot propulsion. Most favorable surface morphology was obtained by the polymerization using a low monomer concentration in the presence of proper surfactant $(100 \mathrm{mM})$. The improvement of the surface quality in presence of surfactant like long-chain alkyl sulfonate groups can be mainly attributed to the decreased oxidation potential of the monomer under the same conditions. ${ }^{52}$ Furthermore, the addition of surfactant has improved both the solubility of the monomer and the morphological properties of the polymer because of its dopant anion role in the polymer chain structure. ${ }^{53,54}$ Thus, the surfactant/monomer ratio is also an important parameter for controlling the surface morphology and physical properties in PEDOT/Pt microtubes for diverse applications.

Another variable that had an effect on the yield, conductivity and morphology of the polymer microtube structure and growth was the nature of the electrolyte. For example, we compared the growth of PEDOT/Pt microtubes in the presence of lithium perchlorate $\left(\mathrm{LiClO}_{4}\right)$ and potassium nitrate $\left(\mathrm{KNO}_{3}\right)$ in the same aqueous media. The polymer growth in $\mathrm{LiClO}_{4}$ was not well repeatable under similar polymerization conditions yielding different lengths (not shown). In contrast, $\mathrm{KNO}_{3}$ offered a greatly improved surface morphology, stability and thickness in the PEDOT/Pt microtube structures. The advantage of $\mathrm{KNO}_{3}$ comes from the fact that the charges were consumed more 
rapidly in the presence of nitrate ions than with chlorate ions, indicating the faster deposition of the PEDOT doped with nitrate ions. ${ }^{39}$ In the presence of $\mathrm{KNO}_{3}$, a homogenous polymer (PEDOT) surface was deposited onto membrane template in a short polymerization time.

The use of membranes with different pore diameters allowed for increased control on the size of the mirotubes. For example, with a membrane with a $1 \mu \mathrm{m}$ pore size, one can obtain smaller bots that are half the size of usual microtubes. SI Fig. 2 shows a single (a) and multiple (b) smaller PEDOT/Pt microtubes with lengths of only $4 \mu \mathrm{m}$ and opening diameters of less than $800 \mathrm{~nm}$ (half the body length of the normal template based bilayer microbots). The experiments show that the smaller microbots can also achieve a very high speed. As shown in SI Video 2, a small microbot can move at a fast speed of $325 \mu \mathrm{m} \mathrm{s}^{-1}$ in a $4 \%$ $\mathrm{H}_{2} \mathrm{O}_{2}$ (containing $5 \%$ sodium cholate), corresponding to a relative speed of over 80 body lengths $\mathrm{s}^{-1}$.

Previous studies have shown that temperature has a profound influence on the speed of catalytic micro/nanomotors through its effect on the electrochemical reactivity of hydrogen peroxide decomposition. ${ }^{55,56}$ A similar phenomenon was observed here for the new polymer-based microtubes. At a physiological temperature of $37^{\circ} \mathrm{C}$, such template-based PEDOT/Pt microbots can achieve an ultrafast speed of over 1400 body lengths $\mathrm{s}^{-1}$ (10 $\mathrm{mm} \mathrm{s}^{-1}$ in absolute speed), compared to around 500 body lengths $s^{-1}$ in room temperature. Such speed represents the fastest relative speed of any artificial nanomotors reported up to date. ${ }^{57}$ Fig. 3 (and corresponding SI Video 3) shows the time lapse images of one superfast PEDOT/Pt microbot at $37^{\circ} \mathrm{C}$ over a $40 \mathrm{~ms}$ period. The microbot travels at a speed of around $10 \mathrm{~mm} \mathrm{~s}^{-1}$ within $10 \% \mathrm{H}_{2} \mathrm{O}_{2} / 5 \%$ sodium cholate solution, which corresponds to around 1400 body lengths $\mathrm{s}^{-1}$, considering its $7 \mu \mathrm{m}$ body length. Under the same conditions, PANI/Pt based microbots can also achieve a superfast speed of over 730 body lengths $\mathrm{s}^{-1}$ (compared to 350 body lengths $\mathrm{s}^{-1}$ in room temperature, as shown in SI Video 4). We observed that besides increasing temperature, adding hydrazine can also lead to

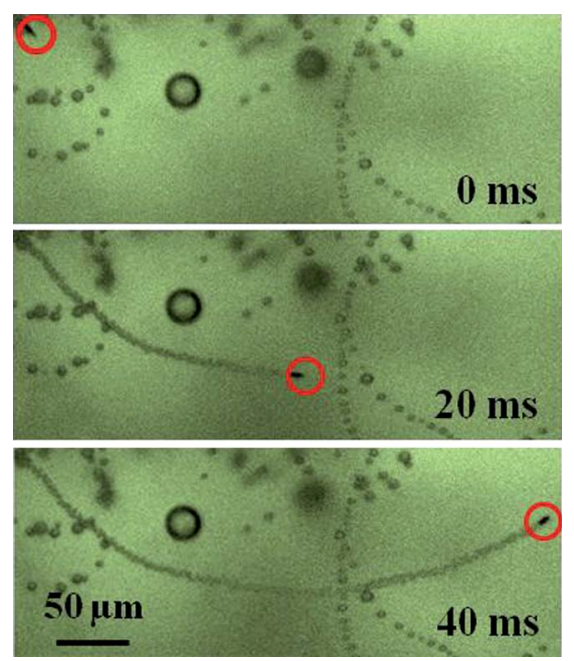

Fig. 3 The ultrafast propulsion (over 1400 body lengths $\mathrm{s}^{-1}$ ) of the PEDOT/Pt microbot in $10 \% \mathrm{H}_{2} \mathrm{O}_{2}$ and $5 \%$ sodium cholate surfactant in physiological temperature of $37^{\circ} \mathrm{C}$. Based on SI Video 3 . a dramatic increase in the speed of catalytic tubular microbots, analogous to the hydrazine effect upon the speed of catalytic nanowire motors. ${ }^{58}$ SI Fig. 3 (and corresponding SI Video 5), show an ultrafast propulsion, at around $5 \mathrm{~mm} \mathrm{~s}^{-1}$ at room temperature - corresponding to around 700 body lengths $\mathrm{s}^{-1}$ - of a PANI/Pt microbot in a $10 \% \mathrm{H}_{2} \mathrm{O}_{2}$ solution spiked with only $0.25 \%$ hydrazine. In fact, the propulsion of the platinum-based microbots using hydrazine fuel alone (without any hydrogen peroxide) has also been observed. For example, SI Video 6, illustrates such motion in a $0.1 \%$ hydrazine solution along with the corresponding $\mathrm{N}_{2}$ bubbles generation; ${ }^{59}$ yet, such propulsion is short-lived and usually lasts only $1-2 \mathrm{~s}$.

While platinum is commonly used as the inner catalytic layer of tubular microbots, we examined the potential use of various alternative catalytic metals. For example, silver is another good catalyst for hydrogen peroxide decomposition, and can therefore be used as the inner catalytic layer of the microbots. Fig. 4A (corresponding to SI Video 7) shows that a PPy/Ag bilayer microbot moving efficiently at a speed of $500 \mu \mathrm{m} \mathrm{s}^{-1}$ in the presence of $15 \%$ hydrogen peroxide and $3 \%$ sodium cholate surfactant. Although the speed of the PPy/Ag microbot is obviously slower than of the platinum-based polymer ones (under same conditions), it is still very fast considering the relative speed of more than 70 body lengths s${ }^{-1}$, compared to common rolledup platinum-based microbots (50 body lengths $\mathrm{s}^{-1}$ in $15 \%$ $\left.\mathrm{H}_{2} \mathrm{O}_{2}\right) \cdot{ }^{17,18}$ Although the silver layer can be partially dissolved in hydrogen peroxide, ${ }^{60,61}$ ultrafast propulsion was observed for over 40 min without considerable speed variations.

Magnetic guidance is essential for the many practical applications of the catalytic micro/nanomotors. As reported before, trilayer microbots with the addition of an inner nickel layer can provide magnetically-guided propulsion, but their preparation requires a 3-step electrodeposition process. ${ }^{25}$ Here we illustrate that a simplified $\mathrm{Pt} / \mathrm{Ni}$ alloy inner layer, instead of separate platinum and nickel layers, can provide simultaneously both the catalytic activity and desired magnetic navigation. Fig. 4B (corresponding to SI Video 8) shows a time lapse image of the propulsion of a PPy/Pt-Ni-alloy microbot in a $10 \%$ hydrogen peroxide solution. The PPy/alloy microbot displays efficient

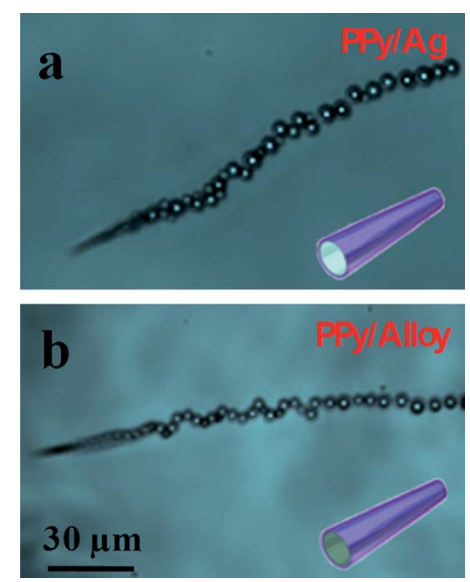

Fig. 4 Propulsion images of PPy-based tubular microbot, based on SI Video 7 and 8. a) PPy/Ag in $15 \% \mathrm{H}_{2} \mathrm{O}_{2}$ and $5 \%$ sodium cholate; b) PPy/ Pt-Ni alloy in $10 \% \mathrm{H}_{2} \mathrm{O}_{2}$ and $5 \%$ sodium cholate. 
propulsion at a high speed of $470 \mu \mathrm{m} \mathrm{s}^{-1}$ (67 body lengths s ${ }^{-1}$ ). However, this is obviously slower than trilayer microbots due to the lower catalytic surface area.

Biocatalytic layers based on immobilized catalase have can serve as attractive alternatives to electrocatalytic metals for propelling peroxide-driven nano/microscale motors. ${ }^{62,63}$ Sanchez et al. showed that catalase-modified rolled-up catalytic microjets can move in $1.5 \%$ hydrogen peroxide at a speed of 10 body lengths $\mathrm{s}^{-1}{ }^{63}$ Similarly, a gold inner layer electrodeposited inside the outer polymeric tube layer can be used to immobilize the catalase biocatalyst. Template electrodeposition of the polymer-Au microtube results in a very rough surface (shown in Fig. 5A) desired for immobilizing large amounts of the enzyme. As illustrated in Fig. 5B (and corresponding to SI Video 9), the resulting biocatalytic bilayer microbots propel favorably in the presence of a low peroxide level $\left(0.5 \% \mathrm{H}_{2} \mathrm{O}_{2}\right)$, at a speed of 8 body lengths s ${ }^{-1}$. $\mathrm{PPy} / \mathrm{Au}$ microtubes (without catalase modification) displayed no directed motion under the same conditions (not shown).

We also examined the possible replacement of the catalytic $\mathrm{Pt}$ layer with inner palladium (Pd) or iridium (Ir) catalytic layers. Unfortunately, it was very difficult to deposit defined palladium or iridium microtubes within the polymer layer. Unlike $\mathrm{Pt}, \mathrm{Ag}$ or $\mathrm{Au}, \mathrm{Pd}$ and Ir usually grow on the top of the tube and block the pore, thus eliminating the tubular bubble propulsion.

Diverse biological applications may require an outer gold layer owing to its facile functionalization and excellent biocompatibility. ${ }^{20-23}$ Here, we demonstrate the template synthesis of $\mathrm{Au} / \mathrm{Pt}$ bilayer microbots coupling these advantages of an outer Au layer with the effective catalytic activity of the inner Pt layer. The $\mathrm{Au}$ outer layer was electrodeposited from a Au plating solution containing $0.1 \mathrm{M} \mathrm{NaNO}_{3}$ using DMSO as an electrolyte (without addition of any other surfactants) ${ }^{64}$ followed by a subsequent deposition of an inner platinum layer. However, one can see clearly that the resulting gold outer surface is relatively rough (SI Fig. 4a). This can be attributed to the interaction of DMSO and the polycarbonate membrane. DMSO slowly dissolves the polycarbonate membranes, making the pores shrink as the microtube is growing. This resulted in a lower yield of viable microtubes and a non-uniform, rough outer layer. The EDX mapping data of SI Fig. 4(b,c) confirmed the presence of the gold and platinum layers. As shown in Fig. 6 (and the corresponding to SI Video 10), such $\mathrm{Au} / \mathrm{Pt}$ bimetallic microbots propel very rapidly at a speed of $1.5 \mathrm{~mm} \mathrm{~s}^{-1}$ in a $10 \% \mathrm{H}_{2} \mathrm{O}_{2}(2.67 \%$ sodium cholate) solution. An improved method for depositing the gold microtube layer should lead to a better microbot performance and yield.

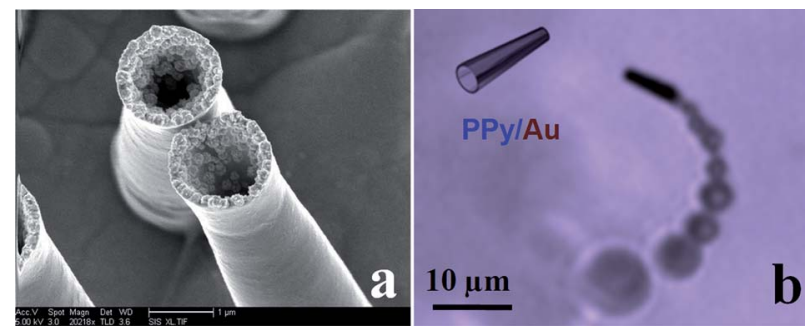

Fig. 5 a) $\mathrm{SEM}$ of $\mathrm{PPy} / \mathrm{Au}_{\text {rough }}$ bilayer micro tubular microbot. b) Timelapse image of biocatalytic propulsion of PPy/Au-catalase in $0.5 \% \mathrm{H}_{2} \mathrm{O}_{2}$ and 2\% sodium cholate, based on SI Video 9.

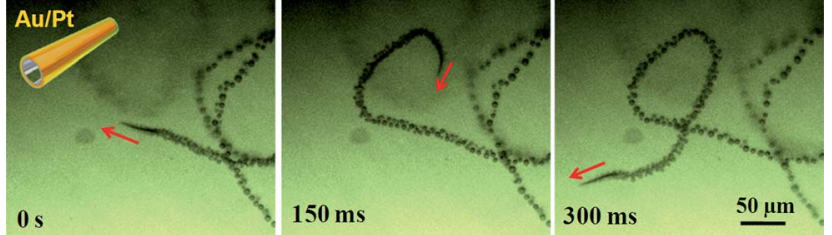

Fig. 6 Ultrafast propulsion of Au/Pt bimetallic tubular microbot in 5\% $\mathrm{H}_{2} \mathrm{O}_{2}$ and $2 \%$ sodium cholate, based on SI Video 10 .

\section{Conclusions}

In conclusion, we evaluated and characterized template-synthesized tubular microbots containing different materials, including various conducting polymers and metals. The critical comparison of different materials and plating conditions used for microbot synthesis had led to the considerate difference on the propulsion and capability for various applications. Template electrodeposition has been shown to be particularly attractive for preparing such new polymer-based catalytic microbots. The influence of the preparation conditions, particularly the electropolymerization conditions, has shown to have a profound effect upon the morphology and propulsion behavior. The new insights into the preparation and composition of template-prepared tubular microbots have facilitated the rational design of highperformance PEDOT/Pt bilayer microbots with a recordbreaking speed of over 1400 body lengths per second at $37^{\circ} \mathrm{C}$. The remarkable speed of the new microbots also reflects their high towing capacity, as desired for diverse biomedical applications. Lower speeds are expected in biological media owing to the increased viscosity. The morphology of the conducting polymer microtube is greatly influenced by the nature and concentration of monomer and of the supporting electrolyte, as well as by the surfactant present. The inherent advantages of easy fabrication of conducting polymer/metal microtubes make them attractive candidates from advanced sensor systems to diverse chemicals such as biomaterials, hydrogen peroxide or methanol fuel cell systems. Moreover, conducting polymer/metal microtubes are inexpensive and versatile because of their properties can be readily modified by the use of a wide range of molecules that can be entrapped or used as dopants. Recently, the polymer microbot have been successfully used for bacteria isolation based on the lectin modifications. ${ }^{65}$ Polymers with different functional groups (such as $-\mathrm{OH}$ or $-\mathrm{COOH}$ ) can be chosen as the outer layer for different applications, hence facilitating different surface functionalization processes. These properties and advantages make the new polymer-based tubular microbots extremely attractive alternatives to current bimetallic microtube systems and hold considerable promise for diverse future applications.

\section{Experimental section}

\section{Synthesis of various tubular microbots}

The various microtubes were prepared using a common template directed electrodeposition protocol. A Cyclopore polycarbonate membrane, containing $2 \mu \mathrm{m}$ diameter conical-shaped micropores (Catalog No 7060-2511; Whatman, Maidstone, U. K.), was employed as the template. A $75 \mathrm{~nm}$ gold film was first sputtered on one side of the porous membrane to serve as working 
electrode using the Denton Discovery 18. The sputter was performed at room temperature under vacuum of $5 \times 10^{-6}$ Torr, DC power $200 \mathrm{~W}$ and flow Ar to $3.1 \mathrm{mT}$. Rotation speed is 65 . Sputter time $90 \mathrm{~s}$. A Pt wire and an $\mathrm{Ag} / \mathrm{AgCl}$ with $3 \mathrm{M} \mathrm{KCl}$ were used as counter and reference electrodes, respectively. The membrane was then assembled in a plating cell with an aluminum foil serving as a contact. Polyaniline microtubes were prepared by modifying the previously described method. ${ }^{25}$ Briefly, aniline and pyrrole (Sigma-Aldrich, St Louis, MO) were distilled before use at a vapor temperature of $100{ }^{\circ} \mathrm{C}$ and a pressure of $13 \mathrm{~mm}$ $\mathrm{Hg}$. Polyaniline (PANI) microtubes were electropolymerized at $+0.80 \mathrm{~V}$ for $0.02 \mathrm{C}$ from a plating solution containing $0.1 \mathrm{M}$ $\mathrm{H}_{2} \mathrm{SO}_{4}, 0.5 \mathrm{M} \mathrm{Na}_{2} \mathrm{SO}_{4}$ and $0.1 \mathrm{M}$ aniline; subsequently, the inner Pt tube was deposited galvanostatically at $-2 \mathrm{~mA}$ for $600 \mathrm{~s}$ from a commercial platinum plating solution (Platinum RTP; Technic Inc, Anaheim, CA).

Poly(3,4-ethylenedioxythiophene) (PEDOT) microtubes were electropolymerized at $+0.80 \mathrm{~V}$ for a charge of $0.06 \mathrm{C}$ from a plating solution containing $15 \mathrm{mM}$ EDOT, $7.5 \mathrm{mM} \mathrm{KNO}_{3}$ and $100 \mathrm{mM}$ sodium dodecyl sulfate (SDS); subsequently, the inner Pt tube was deposited galvanostatically at $-2 \mathrm{~mA}$ for $600 \mathrm{~s}$. The smaller microbots (4 $\mu \mathrm{m}$ in length) were synthesized using a Cyclopore polycarbonate membrane, containing $1 \mu \mathrm{m}$ diameter micropores (Catalog No 7060-2510; Whatman, Maidstone, U. K.) PEDOT microtubes were electropolymerized at $+0.80 \mathrm{~V}$ using a charge of $0.04 \mathrm{C}$ while the corresponding inner platinum layer was deposited galvanostatically at $-2 \mathrm{~mA}$ for $600 \mathrm{~s}$.

Polypyrrole (PPy) microtubes were electropolymerized at $+0.80 \mathrm{~V}$ for a charge of $0.8 \mathrm{C}$ from a plating solution containing $37 \mathrm{mM}$ pyrrole and $7.5 \mathrm{mM} \mathrm{KNO}_{3}$. Subsequently, a metal tube layer was plated inside the polymer layer. Different metallic layers were examined for this purpose. For $\mathrm{PPy} / \mathrm{Pt}$, the inner $\mathrm{Pt}$ tube was deposited galvanostatically at $-2 \mathrm{~mA}$ for $600 \mathrm{~s}$ from a commercial platinum plating solution (Platinum RTP; Technic Inc, Anaheim, CA); for PPy/Ag, silver was plated subsequently at $-0.9 \mathrm{~V}$ (vs. $\mathrm{Ag} / \mathrm{AgCl}$ ) for a total charge of $1 \mathrm{C}$ using a commercial silver plating solution (1025 RTU @ 4.5 Troy/ gallon; Technic Inc., Anaheim, CA); for PPy/Pt-Ni alloy, platinum-nickel alloy is plated at $-1 \mathrm{~V}$ for a total charge of $2 \mathrm{C}$ using a $1: 1$ mixed solution of a commercial platinum solution and a nickel plating solution containing $20 \mathrm{~g} \mathrm{~L}^{-1} \mathrm{NiCl}_{2} \cdot 6 \mathrm{H}_{2} \mathrm{O}$, $515 \mathrm{~g} \mathrm{~L}^{-1} \mathrm{Ni}\left(\mathrm{H}_{2} \mathrm{NSO}_{3}\right)_{2} \cdot 4 \mathrm{H}_{2} \mathrm{O}$, and $20 \mathrm{~g} \mathrm{~L}^{-1} \mathrm{H}_{3} \mathrm{BO}_{3}$; for $\mathrm{PPy}-\mathrm{Au}$, gold is plated at $-0.9 \mathrm{~V}$ for $1 \mathrm{C}$ from a commercial gold plating solution (Orotemp 24 RTU RACK; Technic Inc.).

The sputtered gold layer was completely removed by hand polishing with $3-4 \mu \mathrm{m}$ alumina slurry. The membrane was then dissolved in methylene chloride for $10 \mathrm{~min}$ to completely release the microtubes. The latter were collected by centrifugation at $6000 \mathrm{rpm}$ for $3 \mathrm{~min}$ and washed repeatedly with methylene chloride, followed by ethanol and ultrapure water $(18.2 \mathrm{M} \Omega \mathrm{cm})$, three times of each, with a 3 min centrifugation following each wash. All microtubes were stored in ultrapure water at room temperature when not in use. The microbot preparation method provides a good reproducibility.

\section{Catalase modification}

The inner Au layer from the bilayer microtubes was functionalized with a mixed MUA/MCH monolayer. A solution of $2.5 \mathrm{mM}$
MUA and 7.5 mM MCH was prepared in ethanol. The microbots were incubated in the solution overnight. After rinsing the tubes with ethanol for $5 \mathrm{~min}$, they were transferred to an Eppendorf vial containing a $200 \mu \mathrm{l}$ PBS buffer ( $\mathrm{pH}$ 5.5) solution with the coupling agents 1-Ethyl-3-[3-dimethylaminopropyl] carbodiimide hydrochloride (EDC), N-hydroxylsulfosuccinimide (Sulfo-NHS) at $0.4 \mathrm{M}$ and $0.1 \mathrm{M}$ respectively, and the enzyme catalase $\left(2 \mathrm{mg} \mathrm{mL}^{-1}\right)$. This incubation was carried out $7 \mathrm{~h}$ at $37^{\circ} \mathrm{C}$ and thereafter rinsed with PBS with a $\mathrm{pH}$ of 7.4 and SDS $0.05 \mathrm{wt} \%$ for $15 \mathrm{~min}$ at each step. Finally the microbots were washed repeatedly by centrifugation at $6000 \mathrm{rpm}$ for $3 \mathrm{~min}$ with water for three times to remove extra catalase in solution before testing.

\section{Equipments}

Template electrochemical deposition of microtubes was carried out with a $\mathrm{CHI}$ 661D potentiostat (CH Instruments, Austin, TX). Scanning electron microscopy (SEM) images were obtained with a Phillips XL30 ESEM instrument, using an acceleration potential of $20 \mathrm{kV}$. Mapping analysis was investigated by Oxford EDX attached to SEM instrument and operated by Inca software. An inverted optical microscope (Nikon Instrument Inc. TiS/L100), coupled with a 40x objective, a Photometrics QuantEM 512/SC camera (Roper Scientific, Duluth, GA) and MetaMorph 7.6 software (Molecular Devices, Sunnyvale, CA) were used for capturing movies at a frame rate of 30 frames per sec. The speeds of the microbots were tracked using a Metamorph tracking module and the results were statistically analyzed using Origin software.

\section{Reagents and solutions}

In order to self-propel catalytic microbots, aqueous hydrogen peroxide solutions (Sigma-Aldrich, cat. 95313) with concentrations ranging from $0.5-15.0 \%$ were used as chemical fuels, containing 2-5.0\% (w/v) sodium cholate (Sigma-Aldrich, St Louis, $\mathrm{MO})$ to reduce the surface tension, hence facilitating the microbot propulsion.

\section{Acknowledgements}

This work was supported by the National Science Foundation (Award Number CBET 0853375). A. U. thanks the Fulbright Scholarship program. The authors greatly thank J. Orozco and A. Katzenberg for assisting with the catalase modification and microtube preparation, respectively.

\section{Notes and references}

1 W. F. Paxton, K. C. Kistler, C. C. Olmeda, A. Sen, S. K. St. Angelo, Y. Cao, T. E. Mallouk, P. E. Lammert and V. H. Crespi, J. Am. Chem. Soc., 2004, 126, 13424.

2 S. Fournier-Bidoz, A. C. Arsenault, I. Manners and G. A. Ozin, Chem. Commun., 2005, 441.

3 T. E. Mallouk and A. Sen, Sci. Am., 2009, 300, 72.

4 G. A. Ozin, I. Manners, S. Fournier-Bidoz and A. Arsenault, $A d v$. Mater., 2005, 17, 3011.

5 J. Wang, ACS Nano, 2009, 3, 4.

6 S. Sanchez and M. Pumera, Chem.-Asian J., 2009, 4, 1402.

7 S. J. Ebbens and J. R. Howse, Soft Matter, 2010, 6, 726.

8 L. Zhang, J. J. Abbott, L. Dong, K. E. Peyer, B. E. Kratochvil, H. Zhang, C. Bergeles and B. J. Nelson, Nano Lett., 2009, 9, 3663. 
9 O. S. Pak, W. Gao, J. Wang and E. Lauga, Soft Matter, 2011, 7, 8169.

10 W. Gao, K. M. Manesh, J. Hua, S. Sattayasamitsathit and J. Wang, Small, 2011, 7, 2047.

11 G. Loget and A. Kuhn, J. Am. Chem. Soc., 2010, 132, 15918.

12 R. Liu and A. Sen, J. Am. Chem. Soc., 2011, 133, 20064.

13 G. J. Zhao, T. H. Seah and M. Pumera, Chem.-Eur. J., 2011, 17, 12020.

14 R. A. Pavlick, S. Sengupta, T. McFadden, H. Zhang and A. Sen, Angew. Chem., Int. Ed., 2011, 50, 9374.

15 W. Gao, A. Uygun and J. Wang, J. Am. Chem. Soc., 2012, 134, 897.

16 Y. F. Mei, G. S. Huang, A. A. Solovev, E. Bermúdez Ureña I. Mönch, F. Ding, T. Reindl, R. K. Y. Fu, P. K. Chu and O. G. Schmidt, Adv. Mater., 2008, 20, 4085.

17 A. A. Solovev, Y. F. Mei, E. Bermúdez Ureña, G. S. Huang and O. G. Schmidt, Small, 2009, 5, 1688.

18 Y. F. Mei, A. A. Solovev, S. Sanchez and O. G. Schmidt, Chem. Soc Rev., 2011, 40, 2109.

19 A. A. Solovev, S. Sanchez, M. Pumera, Y. F. Mei and O. G. Schmidt, Adv. Funct. Mater., 2010, 20, 2430.

20 S. Campuzano, D. Kagan, J. Orozco and J. Wang, Analyst, 2011, 136, 4621.

21 S. Balasubramanian, D. Kagan, C. M. Hu, S. Campuzano, M. J. Lobo-Castañon, N. Lim, D. Y. Kang, M. Zimmerman, L. Zhang and J. Wang, Angew. Chem., Int. Ed., 2011, 50, 4161.

22 D. Kagan, S. Campuzano, S. Salasubramanian, F. Kuralay, G. U. Flechsig and J. Wang, Nano Lett., 2011, 11, 2083.

23 J. Orozco, S. Campuzano, D. Kagan, M. Zhou, W. Gao and J. Wang, Anal. Chem., 2011, 83, 7962.

24 K. M. Manesh, R. Yuan, M. Clark, D. Kagan, S. Balasubramanian and J. Wang, ACS Nano, 2010, 4, 1799.

25 W. Gao, S. Sattayasamitsathit, J. Orozco and J. Wang, J. Am. Chem. Soc., 2011, 133, 11862.

26 T. Darmanin and F. Guittard, J. Am. Chem. Soc., 2011, 133, 15627.

27 Y. Z. Long, M. M. Li, C. Gu, M. Wan, J. L. Duvail, Z. Liu and Z. Fan, Prog. Polym. Sci., 2011, 36, 1415.

28 S. C. Luo, E. M. Ali, N. C. Tansil, H. H. Yu, S. Gao, E. A. B. Kantchev and J. Y. Ying, Langmuir, 2008, 24, 8071.

29 N. K. Guimard, N. Gomez and C. E. Schmidt, Prog. Polym. Sci., 2007, 32, 876 .

30 M. R. Abidian, D. H. Kim and D. C. Martin, Adv. Mater., 2006, 18, 405.

31 J. Sekine, S. C. Luo, S. Wang, B. Zhu, H. R. Tseng and H. H. Yu, Adv. Mater., 2011, 23, 4788.

32 F. Carpi and E. Smela, Biological Applications of Electroactive Polymer Actuators. Wiley, 2009.

33 V. A. Pedrosa, X. Luo, J. Burdick and J. Wang, Small, 2008, 4, 738.

34 L. Xia, Z. X. Wei and M. X. Wan, J. Colloid Interface Sci., 2010, 341,1 .

35 J. M. Pernaut and J. R. Reynolds, J. Phys. Chem. B, 2000, 104, 4080

36 S. I. Cho, W. J. Kwon, S. J. Choi, P. Kim, S. A. Park, J. Kim, S. J. Son, R. Xiao, S. H. Kim and S. B. Lee, Adv. Mater., 2005, 17, 171.
37 S. I. Cho and S. B. Lee, Acc. Chem. Res., 2008, 41, 699.

38 S. I. Cho, W. J. Kwon, S. J. Choi, P. Kim, S. A. Park, J. Kim, S. J. Son, R. Xiao, S. H. Kim and S. B. Lee, Adv. Mater., 2005, 17, 171.

39 C. Zhou, Z. Liu, Y. Yan, X. Du, Y. W. Mai and S. Ringer, Nanoscale Res. Lett., 2011, 6, 364.

40 V. Bajpai, P. He and L. M. Dai, Adv. Funct. Mater., 2004, 14, 145.

41 L. Duic and Z. Mandic, J. Electroanal. Chem., 1992, 335, 207.

42 N. Gospodinova and L. Terlemezyan, Prog. Polym. Sci., 1998, 23, 1443.

43 E. A. Bazzaoui, S. Aeiyach and P. C. Lacaze, Synth. Met., 1996, 83, 159.

44 N. Sakmeche, J. J. Aaron, M. Fall, S. Aeiyach, M. Jouini, J. C. Lacroix and P. C. Lacaze, Chem. Commun., 1996, 2723.

45 Z. Liu, X. Zhang, S. Poyraz, S. P. Surwade and S. K. Manohar, J. Am. Chem. Soc., 2010, 132, 13158.

46 E. Poverenov, M. Li, A. Bitler and M. Bendikov, Chem. Mater., 2010, 22, 4019.

47 K. Jackowska, A. T. Bieguński and M. Tagowska, J. Solid State Electrochem., 2007, 12, 437.

48 J. X. Li, G. S. Huang, M. M. Ye, M. L. Li, R. Liu and Y. F. Mei, Nanoscale, 2011, 3, 5083.

49 C. R. Martin, Science, 1994, 266, 1961.

50 A. I. Melato, A. S. Viana and L. M. Abrantes, Electrochim. Acta, 2008, 54, 590.

51 C. F. Zhou, Z. W. Liu and S. P. Ringer, Synth. Met., 2010, 160, 1636.

52 T. Shimidzu, A. Ohtani, T. Iyoda and K. Honda, J. Electroanal. Chem., 1987, 224, 123.

53 Z. Zhang, Z. Wei and M. Wan, Macromolecules, 2002, 35, 5937.

54 D. Han, G. Yang, J. Song, L. Niu and A. Ivaska, J. Electroanal. Chem., 2007, 602, 24.

55 S. Balasubramanian, D. Kagan, K. M. Manesh, P. Calvo-Marzal, G. U. Flechsig and J. Wang, Small, 2009, 5, 1569.

56 S. Sanchez, A. N. Ananth, V. M. Fomin, M. Viehrig and O. G. Schmidt, J. Am. Chem. Soc., 2011, 133, 14860.

57 W. Gao, S. Sattayasamitsathit and J. Wang, Chem. Rec., 2012, 12, 224.

58 R. Laocharoensuk, J. Burdick and J. Wang, ACS Nano, 2008, 2, 1069.

59 J. A. Harrison and Z. A. Khan, J. Electroanal. Chem., 1970, 28, 131.

$60 \mathrm{~W}$. Gao, S. Sattayasamitsathit, K. M. Manesh, D. Weihs and J. Wang, J. Am. Chem. Soc., 2010, 132, 14403.

61 M. E. Ibele, R. Liu, K. Beiswenger and A. Sen, J. Mater. Chem., 2011, 21, 14410 .

62 Y. Wang, R. M. Hernandez, D. J. Bartlett, J. M. Bingham, T. R. Kline, A. Sen and T. E. Mallouk, Langmuir, 2006, 22, 10451.

63 S. Sanchez, A. A. Solovev, Y. F. Mei and O. G. Schmidt, J. Am. Chem. Soc., 2010, 132, 13144.

64 C. H. Cui, H. H. Li and S. H. Yu, Chem. Commun., 2010, 46, 940.

65 S. Campuzano, J. Orozco, D. Kagan, M. Guix, W. Gao, S. Sattayasamitsathit, J. C. Claussen, A. Merkoçi and J. Wang, Nano Lett., 2012, 12, 396. 